\title{
Dietrich, Christian: Im Schatten August Bebels. Sozialdemokratische Antisemitismusabwehr als Republikschutz 1918-1932, 319 S., Wallstein, Göttingen 2021.
}

\section{Sebastian Elsbach}

Angenommen: 3. Dezember 2021 / Online publiziert: 24. Januar 2022

(C) Der/die Autor(en) 2022

Die Habilitationsschrift des Historikers Christian Dietrich widmet sich dem sozialdemokratischen Anti-Antisemitismus in der Weimarer Republik, wobei eine breitere Verortung in die publizistische und politische „Konstellation“ dieser Parteirichtung erfolgt. Mit dieser aus der Philosophie entlehnten Methodik (S. 25-28) zielt der Autor darauf ab, dass die sozialdemokratischen Aussagen über Antisemitismus ideengeschichtlich verortet werden müssen, aber auch nicht losgelöst von sonstigen innen-, außen- oder kulturpolitischen Erwägungen zu verstehen sind. In diesem Sinne widmet sich Dietrich ausführlich den Themenkreisen des Zionismus und der sogenannten Ostjuden. Die Querverbindungen zwischen diesen drei Fragekomplexen und dem breiteren Kontext der Weimarer (Partei-)Politik bilden die „Konstellation“, in der die sozialdemokratische Antisemitismusabwehr untersucht wird. Demnach waren diese Abwehrreaktionen nicht allein als Schutz der deutschen Juden zu verstehen, sondern hatten immer auch andere Motive, insbesondere den Republikschutz und - hiermit verbunden - den Selbstschutz der Partei beziehungsweise des sozialdemokratischen Milieus. Diese Grundannahme ist durchaus fruchtbar und ähnlich logisch, wie die Ausweitung der Betrachtungen auf den Zionismus und die Ostjudenfrage. Zur Einordnung des sozialdemokratischen Zionismusverständnisses ist es schließlich wichtig zu wissen, dass die Partei ganz unabhängig von der Frage einer jüdischen Kolonisation in Palästina einen klaren Standpunkt gegen den europäischen Kolonialismus entwickelt hatte, der als Teil eines kapitalistischen Imperialismus begriffen wurde (S. 37).

Dietrich setzt sich in einem ersten Hauptteil ausführlich mit den sozialdemokratischen Positionen zum Zionismus im Kaiserreich auseinander. Während Karl Kautsky als wichtigster Theoretiker einer orthodoxen Marxismusauslegung den Kolonialismus primär unter dem Gesichtspunkt der kapitalistischen „Ausbeutung“ betrachtete (S. 39), waren es Vertreter des rechten Parteiflügels, wie Gustav Noske, die in dieser Frage eine offenere Meinung vertraten. Dies hatte mit der Frage einer jüdischen Kolonisation in Palästina zunächst nur am Rande zu tun. Es ging den Rechtssozialisten vor allem um eine vorsichtige Annäherung an die Mehrheitsposition im

Sebastian Elsbach $(\bowtie)$

Friedrich-Schiller-Universität Jena, Jena, Deutschland

E-Mail: sebastian.elsbach@uni-jena.de 
Kaiserreich, zumal andere sozialistische Parteien in Europa sich offen pro-kolonialistisch orientierten (S. $42 \mathrm{ff}$.). Die Sozialistischen Monatshefte als Hauptorgan der sozialdemokratischen Revisionisten zeigten sich jedoch innerhalb dieser „Konstellation“ offen für zionistische Stimmen, die eine jüdische Palästina-Kolonisation erstens als Beitrag zur Lösung des Antisemitismus-Problems in Osteuropa betrachteten und zweitens eine wirtschaftliche Aufwertung des als rückständig betrachteten Palästinas versprachen (S. 46f.). Solche Wortmeldungen in einem einflussreichen Publikationsorgan sorgten wiederum dafür, dass der Widerstand gegen zionistische Positionen langsam aufgeweicht und das Problem insgesamt in der Arbeiterbewegung stärker diskutiert wurde. Das Modell eines „Arbeiterzionismus“ fand daraufhin insbesondere in der USPD Unterstützung (S. 72f.).

Auf die Welle des Antisemitismus, die nach Kriegsniederlage und Novemberrevolution über Deutschland schwappte, reagierte man in der Sozialdemokratie jedoch weiterhin mit dem von August Bebel geprägten Argument, demnach der Antisemitismus als Instrument reaktionärer Kräfte zur Verwirrung der Bevölkerung zu betrachten sei (S. 96 ff.). Diese Vorprägung trug wesentlich dazu bei, dass beide sozialdemokratischen Parteien dem Antisemitismus entschlossen entgegentraten und vielfältige publizistische Strategien zu dessen Eindämmung entwickelten, wie Dietrich in einem umfangreichen zweiten Hauptteil zeigen kann. Diese reichte von klassischen Aufklärungsschriften, parlamentarischen Aktionen und administrativen Maßnahmen bis hin zur Satire. Wie der Titel der Arbeit schon andeutet, gelang es aber nur bedingt aus dem „Schatten Bebels“ herauszutreten und neue Gegenstrategien wider den radikalisierten NS-Antisemitismus zu finden. Während Bebels Diktum bei der Auseinandersetzung mit dem Antisemitismus der Deutschnationalen durchaus hilfreich war, passte es Ende der 1920er Jahre nicht mehr so recht in die Zeit (S. 288). Ein cleverer Spruch aus dem Wahren Jacob brachte dies auf den Punkt: „Einst war der Antisemitismus <der Sozialismus der dummen Kerle>: Jetzt ist es der Terrorismus der feinen Kerle." (S. 200). Die SPD musste bei ihrer Bekämpfung der NSDAP nun gezwungenermaßen verstärkt auf die Straße ausweichen und Dietrich wendet sich unter diesem Gesichtspunkt folgerichtig dem Reichsbanner Schwarz-Rot-Gold zu. Der wichtige Beitrag des Reichsbanners zur Bekämpfung des Antisemitismus wurde bereits verschiedentlich festgestellt. Dietrich beschränkt sich in diesem Teil seiner Arbeit aber nicht auf ideen- oder parlamentsgeschichtliche Betrachtung, sondern präsentiert die Ergebnisse von lokalen Archivrecherchen (S. 264-283). So kann er den Befund erhärten, dass es besonders zu Beginn der 1930er Jahre eine intensive Kooperation zwischen dem Reichsbanner und jüdischen Abwehrorganisationen wie dem Centralverein und dem Reichsbund jüdischer Frontsoldaten gegeben hat.

Der flüssig geschriebenen Arbeit Dietrichs gelingt es so einen vielschichtigen Themenkomplex aufzuarbeiten, der für Verständnis sozialdemokratischer Politik in der Zwischenkriegszeit von wesentlicher Bedeutung ist und auch Kenner_innen des Faches werden die Studie mit einem Erkenntnisgewinn lesen können. Deutliche Schwächen leistet sich Dietrich nicht, auch wenn eine größere Zuspitzung der Thesen nicht geschadet hätte.

Funding Open Access funding enabled and organized by Projekt DEAL. 
Open Access Dieser Artikel wird unter der Creative Commons Namensnennung 4.0 International Lizenz veröffentlicht, welche die Nutzung, Vervielfältigung, Bearbeitung, Verbreitung und Wiedergabe in jeglichem Medium und Format erlaubt, sofern Sie den/die ursprünglichen Autor(en) und die Quelle ordnungsgemäß nennen, einen Link zur Creative Commons Lizenz beifügen und angeben, ob Änderungen vorgenommen wurden.

Die in diesem Artikel enthaltenen Bilder und sonstiges Drittmaterial unterliegen ebenfalls der genannten Creative Commons Lizenz, sofern sich aus der Abbildungslegende nichts anderes ergibt. Sofern das betreffende Material nicht unter der genannten Creative Commons Lizenz steht und die betreffende Handlung nicht nach gesetzlichen Vorschriften erlaubt ist, ist für die oben aufgeführten Weiterverwendungen des Materials die Einwilligung des jeweiligen Rechteinhabers einzuholen.

Weitere Details zur Lizenz entnehmen Sie bitte der Lizenzinformation auf http://creativecommons.org/ licenses/by/4.0/deed.de.

\section{Osterloh, Jörg: ,Ausschaltung der Juden und des jüdischen Geistes". Nationalsozialistische Kulturpolitik 1920-1945 (Wissenschaftliche Reihe des Fritz-Bauer-Instituts, Bd. 34), 644 S., Campus, Frankfurt a. M. u.a. 2020.}

\section{Klaus-Peter Friedrich}

Angenommen: 23. November 2021 / Online publiziert: 8. Januar 2022

(C) Gesellschaft zur wissenschaftlichen Förderung politischer Literatur e.V. and the Author(s) 2021

Seit dem Hohenzollern'schen Kaiserreich wurde die „Verjudung“ des deutschen Kulturlebens beklagt und ihr der Kampf angesagt. Lautstark erhoben mehr oder weniger einflussreiche Antisemiten die Forderung, alle Juden und alles ,Jüdische“ daraus zu entfernen. Die 1920 gegründete NSDAP wollte dann die „Ausschaltung der Juden und des jüdischen Geistes“ in der deutschen Kultur, Presse und im Verlagswesen erreichen. Was bis 1945 daraus wurde, hat Jörg Osterloh nun in seiner Studie über die nationalsozialistische Kulturpolitik ,,auf Basis der Forschungsliteratur als auch der Quellen umfassend“" beschrieben (S. 33). Er untersucht damit erstmals systematisch die Beseitigung von jüdischen Deutschen aus Kunst, Musik, Literatur, Theater und Film - bis hin zu ihrer Vertreibung und/oder Ermordung. Neben den reichsweiten Geschehnissen stehen dabei wiederholt landespolitische Entwicklungen in den Ländern Thüringen, Braunschweig und Oldenburg im Mittelpunkt, wo Natio-

Klaus-Peter Friedrich $(\bowtie)$

Marburg, Deutschland

E-Mail: klaus-peter.friedrich@gmx.de 